Number of pages: 16

Number of references: 30

Number of figures: 9

Number of tables: 1

\title{
Equivalent comfort contours for vertical seat vibration: Effect of vibration magnitude and backrest inclination
}

Authors:

Bazil Basri and Michael J. Griffin

Affiliations:

Human Factors Research Unit

Institute of Sound and Vibration Research

University of Southampton

Southampton, SO17 1BJ

England

Corresponding author:

Professor Michael J. Griffin

Tel: +44 (0) 2380592277

Fax: $+44(0) 2380592927$

Email: M.J.Griffin@soton.ac.uk 


\begin{abstract}
This study determined how backrest inclination and the frequency and magnitude of vertical seat vibration influence vibration discomfort. Subjects experienced vertical seat vibration at frequencies in the range 2.5 to $25 \mathrm{~Hz}$ at vibration magnitudes in the range 0.016 to $2.0 \mathrm{~ms}^{-2}$ r.m.s. Equivalent comfort contours were determined with five backrest conditions: no backrest, and with a stationary backrest inclined at $0^{\circ}$ (upright), $30^{\circ}, 60^{\circ}$ and $90^{\circ}$. Within all conditions, the frequency of greatest sensitivity to acceleration decreased with increasing vibration magnitude. Compared to an upright backrest, around the main resonance of the body the vibration magnitudes required to cause similar discomfort were $100 \%$ greater with $60^{\circ}$ and $90^{\circ}$ backrest inclinations and $50 \%$ greater with a $30^{\circ}$ backrest inclination. It is concluded that no single frequency weighting provides an accurate prediction of the discomfort caused by vertical seat vibration at all magnitudes and with all backrest conditions.
\end{abstract}

\title{
Relevance of the findings for ergonomics practice
}

Vertical seat vibration is a main cause of vibration discomfort for drivers and passengers of road vehicles. A frequency weighting has been standardised for the evaluation of vertical seat vibration when sitting upright but it was not known whether this weighting is suitable for the reclined sitting postures often adopted during travel.

Keywords: Backrest angle; ride comfort; frequency weighting. 


\section{INTRODUCTION}

Understanding of how the discomfort caused by whole-body vibration depends on the frequency and direction of vibration has provided a foundation for frequency weightings used in the evaluation of vehicle ride comfort and the dynamic performance of seating. Vehicle vibration enters the body through the seat, the back, and the feet and it is assumed that by using frequency weightings appropriate for each interface between the body and the seat it is possible to predict the vibration discomfort. Frequency weightings have been developed for vibration at these three locations for an upright seated posture. There has been little study of vibration discomfort in a semi-supine posture, leading to doubt over the suitability of current frequency weightings when evaluating vibration in the reclined sitting postures common when travelling. Experiments have examined the suitability of the current frequency weightings for evaluating the vibration of inclined backrests in a direction normal to the back (i.e. $x$-axis vibration of the back; Basri and Griffin, 2011a) and in a direction parallel to the back (i.e. z-axis vibration of the back; Basri and Griffin, 2011b). The present study is concerned with the effect of backrest inclination on frequency weightings for evaluating vertical seat vibration.

Vertical seat vibration is often the dominant cause of vibration discomfort in road vehicles when the vibration is evaluated in accord with current standards (BS 6841, 1987; ISO 26311, 1997). For predicting the discomfort caused by vertical seat vibration, the frequency weighting $W_{\mathrm{b}}$ is usually recommended. It was developed from consideration of equivalent comfort contours obtained from the vertical vibration of subjects sitting upright without a backrest over the frequency range 1 to $63 \mathrm{~Hz}$ (Griffin et al., 1982) and those obtained with a backrest at frequencies from 0.5 to $5 \mathrm{~Hz}$ (Corbridge and Griffin, 1986). The method of constant stimuli was used with the reference vibration at two magnitudes $\left(0.5\right.$ and $1.25 \mathrm{~ms}^{-2}$ r.m.s. at $10 \mathrm{~Hz}$ in Griffin et al., 1982; 0.25 and $0.75 \mathrm{~ms}^{-2}$ r.m.s. at $2 \mathrm{~Hz}$ in Corbridge and Griffin, 1986) so as to determine the effects of both the frequency and the magnitude of vibration on the equivalent comfort contours. The frequency-dependence of the contours was reasonably consistent with that obtained for the vertical vibration of upright seated people with no backrest as reported in earlier studies (Miwa, 1967; Jones and Saunders. 1972; Dupuis et. al, 1972; Griffin, 1976). The frequency at which acceleration caused greatest discomfort was around about $5 \mathrm{~Hz}$, similar to the resonance frequency evident in the vertical apparent mass (e.g. Fairley and Griffin, 1989). Using magnitude estimation and a wider range of frequencies and magnitudes of vibration, Morioka and Griffin (2006) found a similar frequency-dependence of the equivalent comfort contours but observed that the shapes of the equivalent comfort contours depended on the vibration magnitude: the region of greatest sensitivity to vibration acceleration reduced from somewhere in the range 5 to 10 
$\mathrm{Hz}$ to around $4 \mathrm{~Hz}$ as the vibration magnitude increased from about 0.05 to $1.0 \mathrm{~ms}^{-2}$ r.m.s. This is consistent with a nonlinearity in the apparent mass of the body in which the resonance frequencies reduce from about 6 to $4 \mathrm{~Hz}$ as the magnitude of excitation increases from 0.125 to $2.0 \mathrm{~ms}^{-2}$ r.m.s. (e.g. Fairley and Griffin, 1989; Matsumoto and Griffin, 2002). As a consequence of the nonlinearity in the subjective responses, at high magnitudes the $W_{\mathrm{b}}$ weighting tended to either overestimate discomfort at frequencies away from the principal body resonance or underestimate the discomfort caused by frequencies close to the resonance.

It has been reported that the resonance frequencies of the vertical apparent mass of the seated human body measured at the seat increase from around $5 \mathrm{~Hz}$ to 6.35 or $7.5 \mathrm{~Hz}$ as a rigid backrest is inclined from $0^{\circ}$ to $30^{\circ}$ (Shibata and Maeda, 2009; Toward and Griffin, 2009). The resonance in the vertical apparent mass of a fully supine body has been found around 5.5 to $7.0 \mathrm{~Hz}$, with the resonance increasing to around 7.0 to $9.4 \mathrm{~Hz}$ as the area of backrest contact increased in a semi-supine posture (Huang and Griffin, 2008). The primary peak in the transmissibility of vibration to the sternum increased from around 7.0 to $10.2 \mathrm{~Hz}$ and from 9.4 to $10.9 \mathrm{~Hz}$ in fully supine and semi-supine postures, respectively (Huang and Griffin, 2009). With evidence of increased resonance frequencies in the biodynamic responses with increasing backrest inclination (i.e., from upright backrest $\left(0^{\circ}\right)$ to $30^{\circ}$ and $90^{\circ}$ (supine) as mentioned above), it seems likely that the frequency-dependence of vibration discomfort caused by vertical seat vibration will also depend on backrest inclination.

Performance of a tracking task and a choice reaction time task has been investigated with five backrest inclinations from upright to recumbent (i.e., supine) during exposure to vertical whole-body vibration ( $2 \mathrm{~ms}^{-2}$ r.m.s. in one of three frequency bands: 2 to $4 \mathrm{~Hz}, 8$ to $14 \mathrm{~Hz}$, or 14 to $20 \mathrm{~Hz}$ ) (Paddan et al., 2012). It was concluded that different tasks, displays, controls, vibration magnitudes, and vibration durations could produce different results, but for the conditions investigated, performance during vibration was not dependent on backrest inclination, except with a $67.5^{\circ}$ inclination, where there was poorer performance with the tracking task.

The main objective of the present study was to advance understanding of the vibration discomfort caused by vertical seat vibration when sitting with a reclined backrest and to assess the suitability of current frequency weightings. To facilitate a comparison with previous findings, equivalent comfort contours were determined for vertical seat vibration with no backrest as well as with a backrest inclined by $0^{\circ}$ (i.e. upright), $30^{\circ}, 60^{\circ}$ and $90^{\circ}$ (i.e. recumbent). Additionally, the relative discomfort caused by vertical seat vibration with these five backrest conditions was determined and the location of vibration discomfort in the body 
was investigated. It was hypothesised that the frequency-dependence of the discomfort caused by vertical seat vibration would change with backrest inclination and that the shapes of equivalent comfort contours would depend on the magnitude of vibration, especially around the frequency of body resonances.

\section{METHOD}

\subsection{Apparatus}

A seat was designed to allow vertical seat vibration with five backrest conditions (no backrest and with the backrest at four different inclinations). A Derritron VP85 vibrator was connected to a rigid flat seat with an adjustable stationary backrest and a stationary footrest (or horizontal support for the calves when fully reclined) (Figure 1). The backrest was adjustable to inclinations of $0^{\circ}, 30^{\circ}, 60^{\circ}$, or $90^{\circ}$ (fully recumbent) and could be detached for the no backrest condition. With each backrest condition, the apparatus was adjusted according to a comfortable sitting posture for a $50^{\text {th }}$ percentile British male aged 19 to 45 years (Pheasant, 1990). The positions were achieved using an $\mathrm{H}$-point manikin with knee and ankle angles set to $120^{\circ}$ and $100^{\circ}$, respectively. With the backrest inclined at $90^{\circ}$, subjects lay flat on their backs with their calves supported. A headrest padded with $5-\mathrm{cm}$ thick foam was attached to the backrest and positioned according to the subject height in all conditions except when there was no backrest and when the backrest was upright. The contact between the back and the backrest was predominantly at the upper back, with no contact around the lumbar and pelvic region. The supports for the back, head and calves were covered with 1-mm thick neoprene rubber to provide friction between the supports and the body.

\section{FIGURE 1 ABOUT HERE}

A cylindrical wooden handle (3.18-cm diameter and 12-cm long) was attached to the table of a vertically-orientated Derritron VP4 vibrator supported on a height-adjustable stand. The location and height of the handle were adjusted for each subject so as to maintain the posture of the hand at a similar and comfortable position with all five backrest conditions. The upper-arm and fore-arm were maintained with a slight bend (about 90 to $120^{\circ}$ degrees) to avoid 'locking' at the elbow, and thereby reducing the transmission of vibration to the shoulders, so that discomfort caused by vibration of the handle was localized around the hand (Figure 2). 


\subsection{Vibration and signal generation}

The vibration was generated and sampled using HVLab software (version 3.81) and output via a digital-to-analogue converter (PCL-818) at 1000 samples per second after low-pass filtering at $40 \mathrm{~Hz}$.

Single-axis piezo-resistive accelerometers (Entran Model EGCSY-240D-10) were attached underneath the seat and at the base of the handle. Signals from the accelerometers were filtered at $40 \mathrm{~Hz}$ (via a Techfilter anti-aliasing filter) and then sampled at 1000 samples per second.

The background vibration on the seat was predominantly caused by electrical noise at $50 \mathrm{~Hz}$ and was imperceptible at a magnitude less than $0.012 \mathrm{~ms}^{-2}$ r.m.s.

\subsection{Vibration stimuli}

The vibration stimuli were 2-second duration sinusoids with 0.25 -second cosine-tapering at the start and end.

In Part 1, subjects judged the vibration discomfort caused by various frequencies and magnitudes of vertical seat vibration: they compared the discomfort caused by 'test' vibrations relative to a 'reference' vibration $\left(0.20 \mathrm{~ms}^{-2}\right.$ r.m.s. $8-\mathrm{Hz}$ vertical seat vibration). The reference vibration was at the middle of the range of frequencies and at the middle of the range of magnitudes used for the test vibration. There were 99 test vibrations from an array of 11 frequencies (the preferred one-third octave centre frequencies from 2.5 to $25 \mathrm{~Hz}$ ) and 9 magnitudes (from $0.016 \mathrm{~ms}^{-2}$ r.m.s. at $2.5 \mathrm{~Hz}$, increasing by $6 \mathrm{~dB}$ per octave across the frequency range, and at nine levels increasing in 3-dB steps). The dominant discomfort associated with the vertical vibration in many forms of transport is produced by vibration within these ranges of frequency and magnitude (e.g., road, off-road and rail vehicles, helicopters).

In Part 2, the same nine 8- $\mathrm{Hz}$ vertical seat vibration test stimuli presented in Part 1 were judged relative to the discomfort caused by $2.0 \mathrm{~ms}^{-2}$ r.m.s. $8-\mathrm{Hz}$ vertical hand vibration. This made it possible to quantify any differences in the vibration discomfort caused by the reference frequency due to the different backrest conditions (see below).

In Part 3, the test stimuli were two magnitudes of vertical seat vibration at each frequency: the middle magnitudes and the greatest magnitudes used in Part 1. 


\section{$2.4 \quad$ Procedure}

The experiment was conducted in five sessions corresponding to the five backrest conditions (i.e. no backrest, and the backrest inclined at $0^{\circ}, 30^{\circ}, 60^{\circ}$, and $90^{\circ}$ ). Each subject completed all five sessions within three days, with one session on the first day followed by two sessions on the second day and the remaining two sessions on the final day. Each session lasted less than 30 minutes. The order of the session was balanced between subjects. In the first session, subjects had a short exercise judging the apparent length of lines relative to the length of a reference line. They also practiced judging vibration before commencing the experiment, so as to confirm they understood the magnitude estimation method. On the second and final day, subjects were provided with a 5 to 10 minutes break between sessions.

Each session comprised three parts corresponding to three psychophysical tests:

Part 1: Equivalent comfort contours within backrest condition,

Part 2: Relative discomfort between backrest conditions, and

Part 3: Location of discomfort.

Subjects were requested to sit on the seat with their backs leaning comfortably against the backrest and their hands resting on their laps, or folded together on top of their stomach when the backrest was inclined to $60^{\circ}$ and $90^{\circ}$ (recumbent). In the no backrest condition and in the upright backrest condition, there was no support for the head and the subjects were requested to sit with comfortable upright postures throughout. When the backrest was present, the subjects were asked to maintain contact between their upper back and the upright backrest. For backrest inclinations of $0^{\circ}, 30^{\circ}$, and $60^{\circ}$, the feet were supported, whereas when recumbent (at $90^{\circ}$ ), the calves were supported.

Subjects were blindfolded to avoid seeing their body movement and they wore headphones presenting a masking white noise at $70 \mathrm{~dB}(\mathrm{~A})$. In Part 3, the blindfold was removed to enable subjects to see a body map placed in front of them. The experimenter and subjects were provided with separate emergency stop buttons.

\subsubsection{Equivalent comfort contours within backrest condition: Part 1}

Subjects were requested to provide a magnitude estimate, $\psi$, for each test stimulus at an acceleration magnitude, $\varphi$. The estimation was based on their judgement of the discomfort caused by each test stimulus relative to that caused by the reference stimulus $(8-\mathrm{Hz}$ at 0.20 $\mathrm{ms}^{-2}$ r.m.s.), assumed to correspond to a magnitude estimate of 100 . The reference and test stimuli were vertical seat vibrations presented in pairs separated by 1 -second pauses. The 
frequencies and magnitudes of the test stimuli were presented in a different randomized order for each subject. This part was completed in approximately 20 minutes.

\subsubsection{Relative discomfort between backrest conditions: Part 2}

The discomfort caused by the reference vibration used in Part 1 (i.e., 0.2 ms $^{-2}$ r.m.s. $8-\mathrm{Hz}$ vertical seat vibration) might not be the same with all backrest inclinations, so an 'adjustment' of the reference was required before the relative discomfort between backrest inclinations could be determined. With each backrest condition, subjects therefore judged the discomfort caused by the nine levels of $8-\mathrm{Hz}$ vertical seat vibration presented in Part 1 relative to the discomfort caused by the 'common reference' (i.e., $8-\mathrm{Hz}$ vertical hand vibration at $2.0 \mathrm{~ms}^{-2}$ r.m.s.). This part was completed in approximately 3 minutes.

\subsubsection{Location of discomfort: Part 3}

The middle magnitude and the greatest magnitude of vertical seat vibration at each frequency in Part 1 were presented again in a randomised order. After experiencing each vibration, subjects indicated the location where there was most discomfort in their body according to a body map displayed in front of them. This part was completed in approximately 5 minutes.

\subsection{Subjects}

Using a within-subject experimental design, twelve healthy male subjects participated in all five sessions of the experiment. Subjects had a mean age of 27.8 years (SD: 5.2 ), a mean stature of $1.72 \mathrm{~m}$ (SD: 5.0), and a mean weight of $65.6 \mathrm{~kg}$ (SD: 10.3). Subjects were students and staff of the University of Southampton with no history of any serious illness, injury, or disability that might affect their judgement of vibration sensations. The experiment was approved by the Human Experimentation Safety Ethics Committee of the Institute of Sound and Vibration Research at the University of Southampton. All subjects gave their voluntary consent prior to the start of their first session on each day.

\section{RESULTS}

\subsection{Equivalent comfort contours within backrest condition (Part 1)}

\subsubsection{Rates of growth of discomfort}

According to Stevens' power law (Stevens, 1975), the magnitude estimates of vibration discomfort, $\psi$, will be related to the vibration magnitude, $\varphi$, by the relation: 
So,

$$
\psi=k \varphi^{n}
$$

Equation 1

$\log _{10} \psi=n \log _{10} \varphi+\log _{10} k$

Equation 2

Individual values for the rate of growth of discomfort, $n$, and the constant, $k$, were determined from the slopes and intercepts of linear least squares regressions between $\log _{10} \psi$ and $\log _{10} \varphi$.

The rates of growth of discomfort, $n$, associated with vertical seat vibration within each of the five backrest conditions (no backrest and backrest inclined 0, 30, 60 and $90^{\circ}$ ) were strongly dependent on the frequency of vibration (Figure $3 ; p<0.001$, Friedman).

\section{FIGURE 3 ABOUT HERE}

The rate of growth of discomfort caused by vertical seat vibration did not differ significantly across backrest conditions at any frequency ( $p>0.05$, Friedman), except at $5 \mathrm{~Hz}(p=0.031)$ and at $6.3 \mathrm{~Hz}(p=0.01)$. The findings were similar when excluding the condition with no backrest: the effect of backrest inclination was not significant at any frequency except at 5 $\mathrm{Hz}(p=0.037)$ and $6.3 \mathrm{~Hz}(p=0.003)$. Six pairwise comparisons performed between the four backrest inclinations at these two frequencies with an adjusted significant level $(p=0.05 / 6=$ 0.008 ), showed the rate of growth of discomfort of vertical seat vibration only differed significantly between backrest inclinations of $60^{\circ}$ and $0^{\circ}$ (upright) with $6.3-\mathrm{Hz}$ vibration (Wilcoxon, $p=0.006$ ).

\subsubsection{Equivalent comfort contours within backrest condition}

Individual equivalent comfort contours were calculated at nine sensation magnitudes (from $\psi$ $=40$ to 250 , relative to 100 with $0.20 \mathrm{~ms}^{-2}$ r.m.s. of $8-\mathrm{Hz}$ vertical seat vibration) using Equation 1 and the individual $n$ and $k$ values at each frequency. Median equivalent comfort contours were constructed from the medians of the 12 individual equivalent comfort contours at each of these nine sensation magnitudes.

As expected from the dependence of the rate of growth of discomfort on the frequency of vibration, the shapes of the equivalent comfort contours varied systematically with the vibration magnitude within all backrest conditions (Figure 4).

TABLE 1 and FIGURE 4 ABOUT HERE

\subsection{Relative discomfort between backrest conditions (Part 2)}

Within each backrest condition, an equivalent comfort contour for each subject was constructed at the sensation magnitude of the $8-\mathrm{Hz}$ vertical seat vibration that produced discomfort equivalent to that of the common reference vibration $(8-\mathrm{Hz}$ vertical hand vibration 
at $2.0 \mathrm{~ms}^{-2}$ r.m.s.), using individual $n$ and $k$ values. The medians of these twelve individual 'rescaled' equivalent comfort contours were determined within each backrest condition to yield equivalent comfort contours having the same reference in all backrest conditions (Figure 5).

\section{FIGURE 5 ABOUT HERE}

The acceleration required at any frequency for discomfort to be equivalent to that caused by the common reference vibration depended on backrest condition at all frequencies less than $12.5 \mathrm{~Hz}$ ( $p<0.05$, Friedman; Figure 5f). When excluding the condition without backrest, the backrest inclination had a significant effect at all frequencies less than $10 \mathrm{~Hz}(p<0.01)$. With the backrest reclined to $90^{\circ}$, the acceleration required to cause discomfort equivalent to the reference vibration was significantly increased by up to $6 \mathrm{~dB}$ at frequencies between 2.5 and $8 \mathrm{~Hz}(p<0.008$, Wilcoxon; Figure $5 a)$ and similarly with the backrest inclined to $60^{\circ}$ at frequencies between 4 and $6.3 \mathrm{~Hz}(p<0.008$, Wilcoxon; Figure $5 \mathrm{~b})$. With the backrest inclined to $30^{\circ}$, the required acceleration was significantly increased by about $3 \mathrm{~dB}$, but only at $5 \mathrm{~Hz}(p<0.008$; Wilcoxon; Figure $5 \mathrm{c})$.

\subsection{Location of discomfort (Part 3)}

As can be seen in Figure 6, with all frequencies and magnitudes and all backrest conditions, subjects mostly felt discomfort in the buttocks or thighs (i.e., the body areas in contact with the seat pan, the source of vibration). However, with no backrest, and with the upright stationary backrest $\left(0^{\circ}\right)$, some subjects also felt discomfort in the back region (i.e. lower and upper back and shoulders) at low frequencies and near the head (i.e., head, neck, or shoulders) at high frequencies, particularly with higher magnitudes of seat vibration. Some subjects also reported discomfort near the head at high frequencies with the backrest inclined at $30^{\circ}$.

As the inclination of the backrest increased (to $30^{\circ}, 60^{\circ}$ and $90^{\circ}$ ), fewer subjects reported discomfort in the buttocks or thighs with low frequency vibration, and more subjects reported discomfort in the back region, particularly with higher magnitudes of seat vibration when recumbent $\left(\right.$ at $\left.90^{\circ}\right)$ (Figure $\left.6 \mathrm{e}\right)$.

\section{FIGURE 6 ABOUT HERE}




\section{DISCUSSION}

\subsection{Effect of frequency}

The equivalent comfort contours obtained without backrest are compared in Figure 7(a) with the findings of previous studies using vertical seat vibration with no backrest and a stationary footrest (e.g. Griffin, 1976; Griffin et. al, 1982; Morioka and Griffin, 2006) or a footrest moving with the seat (e.g. Miwa, 1967; Jones and Saunders, 1972; Dupuis et. al, 1972). The frequencies at which discomfort tends to be greatest (i.e., the least acceleration is required to produce discomfort) vary between studies. In the present study (and some other studies), discomfort caused by frequencies less than about $4 \mathrm{~Hz}$ arose from relative motion between the moving seat pan and the stationary footrest. In the present study this resulted in the majority of subjects (60 to $80 \%$ ) feeling discomfort around the buttocks and thighs. The effect of relative motion between the seat and a stationary footrest on discomfort has previously been shown to be particularly important with low magnitude vibration (Jang and Griffin, 1999; 2000). This explains the difference in low frequency contours between the present study and the contours of Morioka and Griffin who used a contoured seat with no thigh contact. Increased discomfort at frequencies between about 5 and $12.5 \mathrm{~Hz}$ may be explained by body resonances (Fairley and Griffin, 1989).

The equivalent comfort contours obtained with the upright backrest are compared in Figure 7 (b) with the findings of previous studies with upright backrests and footrests moving with the seat (Shoenberger and Harris, 1971; Oborne and Boarer, 1982; Donati et. al, 1983; Corbridge and Griffin, 1986). The frequencies of greatest discomfort reported in these studies are broadly similar, between about 4 or $5 \mathrm{~Hz}$ and $12.5 \mathrm{~Hz}$. The rate at which discomfort reduced with increasing frequency at high frequencies is also similar, as seen in the slopes of the contours. However, the shapes of the contours differ at low frequencies due to relative motion between the seat and the feet and between the seat and the backrest.

In the present study, the equivalent comfort contours obtained with the upright backrest and the backrest inclined by $30^{\circ}$ were similar, but not identical. With the backrest inclined to $30^{\circ}$, there was a significant reduction in discomfort at $5 \mathrm{~Hz}$ (Figure 5c). With the upright backrest, there is a hint of greater sensitivity between 5 and $6.3 \mathrm{~Hz}$, coinciding with a resonance frequency in the vertical apparent mass of the body when sitting with an upright backrest (at $5 \mathrm{~Hz}$ in Shibata and Maeda, 2009; at $5.5 \mathrm{~Hz}$ in Toward and Griffin,2009). With the backrest inclined at $30^{\circ}$, the frequency of greatest discomfort is slightly greater at 6.3 and $8 \mathrm{~Hz}$, consistent with increased resonance frequencies in the apparent mass with $30^{\circ}$-inclined backrests (at 5 and $7.5 \mathrm{~Hz}$ in Shibata and Maeda; at $6.4 \mathrm{~Hz}$ in Toward and Griffin). With the 
backrest inclined to $60^{\circ}$ and $90^{\circ}$ there is a continuing trend towards increased discomfort at higher frequencies with increasing backrest inclination.

The effect of relative motion at low frequencies seems more pronounced with the upright backrest and the $30^{\circ}$ backrest than with no backrest, as seen in steeper slopes in the contours at low frequencies. The percentage of subjects feeling discomfort in their upper back at low frequencies was greater with the upright backrest and the $30^{\circ}$ backrest than with no backrest. Contact between the upper back and the stationary backrest may have increased the effect of relative motion between the moving seat pan and the stationary backrest.

\section{FIGURE 7 ABOUT HERE}

\subsection{Effect of vibration magnitude}

The significant dependence of the rate of growth of discomfort on the frequency of vibration within each backrest condition resulted in strong dependence of equivalent comfort contours on the magnitude of vibration with all backrest conditions. The rate of growth is greater at low frequency than at high frequency, resulting in contours that are closer together at low frequencies than at high frequencies. This can result in dramatic changes so that acceleration at one frequency can cause greater discomfort than at another frequency at low magnitude but less discomfort than the same frequency at high magnitude (Figure 4).

With no backrest, the frequency of greatest discomfort decreased from $8 \mathrm{~Hz}$ as the sensation magnitude increased, consistent with nonlinearity in the apparent mass and transmissibilities to the lumbar spine where resonance frequencies decrease with increases in vibration magnitude from $0.125 \mathrm{~ms}^{-2}$ r.m.s. to $2.0 \mathrm{~ms}^{-2}$ r.m.s. (Matsumoto and Griffin, 2002). Experimental evidence of nonlinearity in the biodynamic responses of the supine body, where voluntary and involuntary muscle activity is expected to have minimal effects, suggests thixotropic properties of the soft tissues may be responsible for reduced stiffness (and a reduced resonance frequency) with higher magnitudes of excitation (Huang and Griffin, 2008; 2009).

With the backrest reclined to $90^{\circ}$ (recumbent), the equivalent comfort contours show dramatic changes with increasing magnitude of vibration (Figure 8). For recumbent subjects, acceleration thresholds have been reported to be greater for the back than for other parts of the body at frequencies less than $30 \mathrm{~Hz}$, but not greatly different from thresholds for the buttocks (Miwa and Yonekawa, 1969). In the present study, at frequencies greater than 4 $\mathrm{Hz}$, the frequency-dependence of discomfort changed from roughly constant jerk (-6 dB per octave) at low magnitudes to roughly constant velocity (+6 dB per octave) at high 
magnitudes. The former is somewhat similar to acceleration thresholds for vertical vibration of recumbent subjects reported in previous studies (Szameitat and Dupuis, 1976; Miwa et. al, 1984; Parsons and Griffin, 1988; Yonekawa et. al, 1999), and the latter similar to a contour representing sensations equivalent to those caused by $0.56 \mathrm{~ms}^{-2}$ r.m.s. $20-\mathrm{Hz}$ vertical vibration in recumbent subjects as determined by Miwa and Yonekawa (1969).

\section{FIGURE 8 ABOUT HERE}

\subsection{Effect of backrest and backrest angle}

The inclination of the stationary backrest had a pronounced effect on the discomfort caused by vertical vibration of the seat, with less discomfort when the backrest was more reclined (Figure $5 a-5 c$ ). Twice as much vibration was required to cause discomfort when the backrest was reclined to $60^{\circ}$ or $90^{\circ}$ and $50 \%$ more vibration was required when reclined to $30^{\circ}$ than with the upright backrest. In comparison, the presence or absence of the stationary vertical backrest had a relatively small effect on the discomfort caused by vertical seat vibration (Figure 5e).

\subsection{Frequency weightings}

British Standard 6841 (1987) advocates the use of frequency weighting $W_{\mathrm{b}}$ for the evaluation of $z$-axis (vertical) vibration of a seat. For each backrest condition, equivalent comfort contours at the nine sensation magnitudes were inverted and normalised to unity at $5 \mathrm{~Hz}$ so as to facilitate comparison with the realisable $W_{\mathrm{b}}$ weighting (Figure 9 ). In all cases, the artefactual influence of relative motion between the vibrating seat and the stationary backrest can be seen at frequencies less than about $4 \mathrm{~Hz}$. With no backrest, at frequencies from 4 to $25 \mathrm{~Hz}$, the $W_{\mathrm{b}}$ weighting seems to provide a reasonable prediction for low sensation magnitudes, but tends to overestimate sensitivity at high frequencies (or underestimate sensitivity around $5 \mathrm{~Hz}$ ) with high magnitudes. As previously reported by Morioka and Griffin (2006), the weighting cannot reflect the nonlinearity in the equivalent comfort contours. There is a similar trend with the upright backrest.

With the backrest reclined to $30^{\circ}$, Figure $9 \mathrm{c}$ shows that the $W_{\mathrm{b}}$ weighting provides a fairly reasonable prediction at low and intermediate magnitudes of vibration, but is less suitable at high magnitudes. With the backrest reclined to $60^{\circ}$ and $90^{\circ}$, there is a contour broadly similar to the $W_{\mathrm{b}}$ weighting, but the large influence of the non-linearity makes it (and any other weighting) inappropriate at some magnitudes.

\section{FIGURE 9 ABOUT HERE}


The application of the results presented here should recognise that other factors may influence the dependence of vibration discomfort on the frequency of vibration and the inclination of a backrest. For example, differences between subjects, including gender and the extremes of age and body size beyond those included in the study may modify the discomfort caused by whole-body vibration.

\section{CONCLUSIONS}

The frequency-dependence of the vibration discomfort caused by vertical seat vibration varies according to the magnitude of the vibration and inclination of the backrest. A single frequency weighting will not provide an optimum evaluation of vertical seat vibration over a wide range of vibration magnitudes and a range of backrest inclinations. With fairly low magnitude vertical seat vibration, the frequency-dependence of discomfort with no backrest, or a backrest inclined to $0^{\circ}$ or $30^{\circ}$, may be represented by the $W_{\mathrm{b}}$ frequency weighting. This weighting will tend to underestimate the discomfort caused by vertical seat vibration at frequencies greater than about $12.5 \mathrm{~Hz}$ if the backrest is reclined to $60^{\circ}$ or $90^{\circ}$.

Relative motion between the vibrating seat and the stationary backrest had a large effect on discomfort at frequencies less than $4 \mathrm{~Hz}$. The effect of the frequency and magnitude of low frequency vertical seat vibration with reclined backrests therefore merits further study in conditions where the backrest and seat move with the same motion.

\section{ACKNOWLEDGEMENT}

This study was conducted with the support from the Ministry of Higher Education of Malaysia and Universiti Teknikal Malaysia, Melaka. The authors are grateful to Dr Miyuki Morioka for technical advice and assistance in providing software used to control the experiment. 


\section{REFERENCES}

Basri, B. and Griffin, M.J., 2011a. The vibration of inclined backrests: perception and discomfort of vibration applied normal to the back in the $x$-axis of the body. Journal of Sound and Vibration, 330 (18-19), 4646-4659.

Basri, B. and Griffin, M.J., 2011b. The vibration of inclined backrests: perception and discomfort of vibration applied parallel to the back in the z-axis of the body. Ergonomics, 54 (12), 1214-1227.

British Standards Institution BS 6841, 1987. Guide to measurement and evaluation of human exposure to whole-body mechanical vibration and repeated shock.

Corbridge, C. and Griffin, M.J. 1986. Vibration and comfort: Vertical and lateral motion in the range $0.5-5.0 \mathrm{~Hz}$. Ergonomics 29, 249-272.

Donati, P., Grosjean, A., Mistrot, P., Rouke, L. 1983. The subjective equivalence of sinusoidal and random whole-body vibration in the sitting position (an experimental study using the 'floating reference vibration' method). Ergonomics, 26 (3), 251-273.

Dupuis, H., Hartung, E., Louda, L., 1972. The effect of random vibrations of a limited frequency band compared with sinusoidal vibrations on human beings, Max-Planck Institute for Agricultural Work and Techniques, Bad Kreuznach.

Fairley, T.E., and Griffin, M.J., 1989. The apparent mass of the seated human body: vertical vibration. Journal of Biomechanics 22 (2) 81-94.

Griffin, M.J., 1976. Subjective equivalence of sinusoidal and random whole-body vibration. Journal of Acoustical Society of America 60 (5) 1140-1145.

Griffin, M.J., Whitham, E.M., Parsons, K.C., 1982. Vibration and comfort I. Translational seat vibration. Ergonomics 25 (7) 603-630.

Huang, Y. and Griffin, M.J., 2008. Nonlinear dual-axis biodynamic response of the semisupine human body during vertical whole-body vibration. Journal of Sound and Vibration, 312 (1-2), 296-315.

Huang, Y. and Griffin, M.J., 2009. Nonlinearity in apparent mass and transmissibility of the supine human body during vertical whole-body vibration. Journal of Sound and Vibration, 324 (1-2), 429-452.

Jang, H.-K, and Griffin, M.J., 1999. The effect of phase of differential vertical vibration at the seat and feet on discomfort. Journal of Sound and Vibration, 223 (5), 785-794.

Jang, H.-K, and Griffin, M.J., 2000. Effect of phase, frequency, magnitude and posture on discomfort associated with differential vertical vibration at the seat and feet. Journal of Sound and Vibration, 229 (2), 273-286.

International Organization of Standardization ISO 2631-1, 1997. Mechanical vibration and shock - evaluation of human exposure to whole-body vibration - part 1: general requirements.

Jones, A.J. and Saunders, D.J., 1972. Effects of postural and methodological changes on equal comfort contours for whole-body, vertical, sinusoidal vibration. Applied Acoustics, 5, 279-299.

Matsumoto, Y. and Griffin, M.J., 2002. Non-linear characteristics in the dynamic responses of seated subjects exposed to vertical whole-body vibration. Journal of Biomechanical Engineering, 124, 527-532.

Miwa, T., 1967. Evaluation methods for vibration effect. Part 1. Measurements of threshold and equal comfort contours of whole body for vertical and horizontal vibrations. Industrial Health, 5, 183-204. 
Miwa, T. and Yonekawa, Y., 1969. Evaluation methods for vibration effect. Part 9. Response to sinusoidal vibration at lying posture. Industrial Health, 7, 116-126.

Miwa, T., Yonekawa, Y., and Kanada, K., 1984. Thresholds of perception of vibration in recumbent person. Journal Acoustical Society of America, 75 (3), 849-854.

Morioka, M. and Griffin, M.J., 2006. Magnitude-dependence of equivalent comfort contours for fore-and-aft, lateral and vertical whole-body vibration. Journal of Sound and Vibration, 298, 755-772.

Oborne, D.J. and Boarer, P.A., 1982. Subjective response to whole-body vibration: The effects of posture. Ergonomics, 25, 673-681.

Paddan, G.S., et. al., 2012. The influence of seat backrest angle on human performance during whole-body vibration. Ergonomics, 55 (1), 114-128.

Parsons, K.C. and Griffin, M.J., 1988. Whole-body vibration perception thresholds. Journal ofSound and Vibration, 121 (2), 237-258.

Pheasant, S., 1990. Bodyspace: Anthropometry, Ergonomics and the Design of Work. 2nd. ed., Taylor \& Francis.

Shibata, N., and Maeda, S., 2009. Determination of backrest inclination based on biodynamic response study for prevention of low back pain. Medical Engineering \& Physics, 32, 577-583.

Shoenberger, R.W., and Harris, C.S., 1971. Psychophysical assessment of whole-body vibration. Human Factors, 13, 41-50.

Stevens, S.S., 1975. Psychophysics: Introduction to its perceptual, neural, and social prospects. New York: John Wiley \& Sons, Inc.

Szameitat, P., and Dupuis, H., 1976. "Über die beeinflussung des liegenden menschen durch mechanische schwingungen". Max-Planck Institut für Landarbeit und Landtechnik. Heft A-76-1, 1-116.

Toward, M.G.R., and Griffin, M.J., 2009. Apparent mass of the human body in the vertical direction: Effect of seat backrest. Journal of Sound and Vibration, 327 (3-5) 657-669.

Yonekawa, Y., Maeda, S., Kanada, K., and Takahashi, Y., 1999. Whole-body vibration perception thresholds of recumbent subjects - Part 1: supine posture. Industrial Health, 37, 398-403. 


\section{FIGURE LEGEND}

Figure 1 Vertical seat vibration with five different backrest conditions: with no backrest (a), with upright backrest or $0^{\circ}$ inclination (b), with backrest inclined at $30^{\circ}$ (c), $60^{\circ}$ (d), and $90^{\circ}$ or recumbent (e).

Figure 2 Similar hand posture and grip on the wooden handle so as to produce equivalent discomfort at the hand with all backrest conditions, achieved by maintaining the angle between upper and lower arm around $90^{\circ}$ to $120^{\circ}$ within each subject and backrest condition: with no backrest (a), with upright backrest or $0^{\circ}$ inclination (b), with backrest inclined at $30^{\circ}(\mathrm{c}), 60^{\circ}(\mathrm{d})$, and $90^{\circ}$ or recumbent (e).

Figure 3 Medians and inter-quartile ranges of the rates of growth of discomfort of vertical seat vibration within each backrest condition (a-e) and comparison on the medians with all backrest conditions (f).

Figure 4 Median equivalent comfort contours for nine magnitude estimates ( $\psi=40$ to 250 ) where 100 corresponds to the discomfort caused by $0.2 \mathrm{~ms}^{-2}$ r.m.s. $8-\mathrm{Hz}$ vertical seat vibration with the same backrest condition: with no backrest (a), with upright backrest or $0^{\circ}$ inclination (b), with backrest inclined at $30^{\circ}(\mathrm{c}), 60^{\circ}(\mathrm{d})$ and $90^{\circ}$ or recumbent (e).

Figure 5 Relative discomfort of vertical seat vibration between backrest conditions: the contours indicate the vibration magnitudes required to produce discomfort equivalent to 2.0 $\mathrm{ms}^{-2}$ r.m.s. of $8-\mathrm{Hz}$ vertical hand vibration (medians from 12 subjects): between upright backrest $\left(0^{\circ}\right)$ and recumbent $\left(90^{\circ}\right)(\mathrm{a})$, between upright backrest and backrest $60^{\circ}(\mathrm{b})$, between upright backrest and backrest $30^{\circ}$ (c), between no backrest and recumbent (d), between no backrest and upright backrest (e), and between all backrest conditions (f).

Figure 6 Principal locations of discomfort in the body caused by the middle and highest magnitude of vibration at each frequency of vertical seat vibration: no backrest (a), upright backrest (b), $30^{\circ}$ inclination (c), $60^{\circ}$ inclination (d), and $90^{\circ}$ inclination (recumbent) (e).

Figure 7 Comparisons of median equivalent comfort contours ( $\psi=40$ to 250 ) for vertical seat vibration with no backrest with contours previously reported for no backrest (a) and upright backrest (b).

Figure 8 Median equivalent comfort contours ( $\psi=40$ to 250) for vertical seat vibration with backrest reclined to $90^{\circ}$ (recumbent) compared with equivalent sensation contours determined for vertical vibration of recumbent persons. Absolute thresholds for the perception of vertical whole-body vibration of recumbent person also shown for studies marked with asterisk.

Figure 9 Median equivalent comfort contours $(\psi=40$ to 250) for vertical seat vibration with all backrest conditions inverted and normalised to unity at $5 \mathrm{~Hz}$ to facilitate comparison with the realisable $W_{\mathrm{b}}$ frequency weighting. 


\section{TABLE LEGEND}

Table 1 Median exponents, $n$, and constants, $k$, for vertical seat vibration with no backrest, and with stationary backrest inclined at $0^{\circ}$ (upright), $30^{\circ}, 60^{\circ}$ and $90^{\circ}$ (recumbent). 


\section{FIGURE 1}
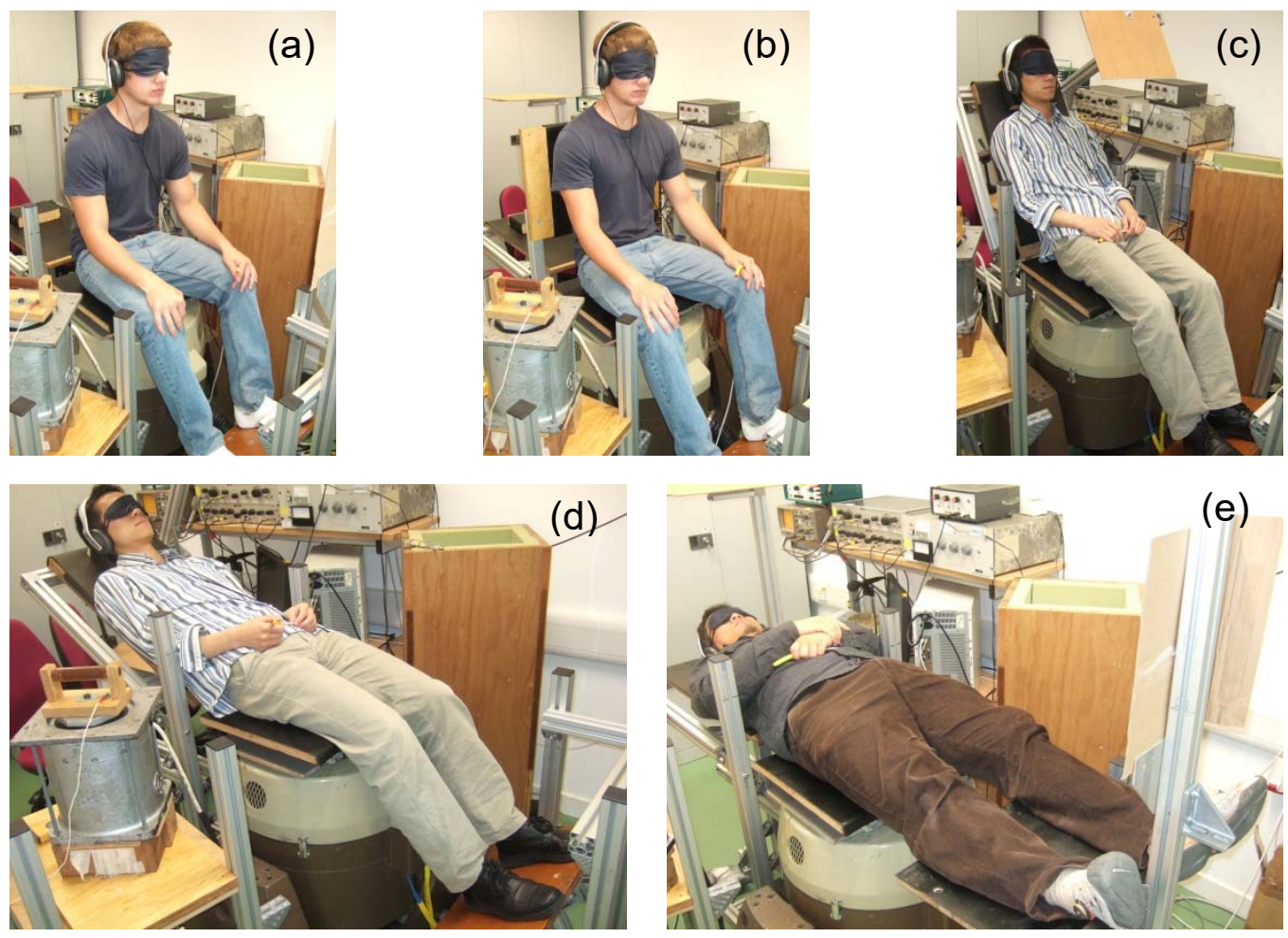


\section{FIGURE 2}
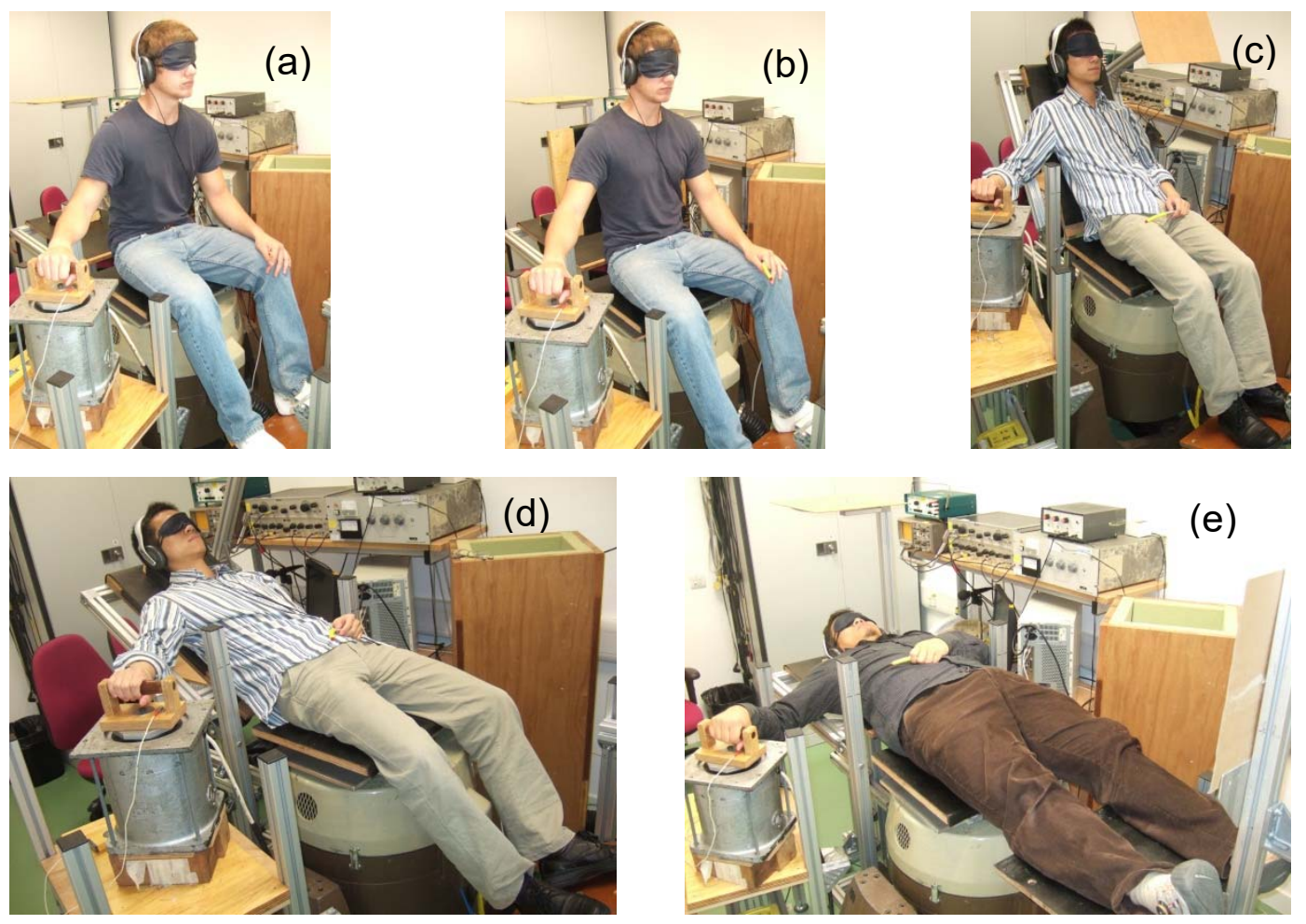


\section{FIGURE 3}

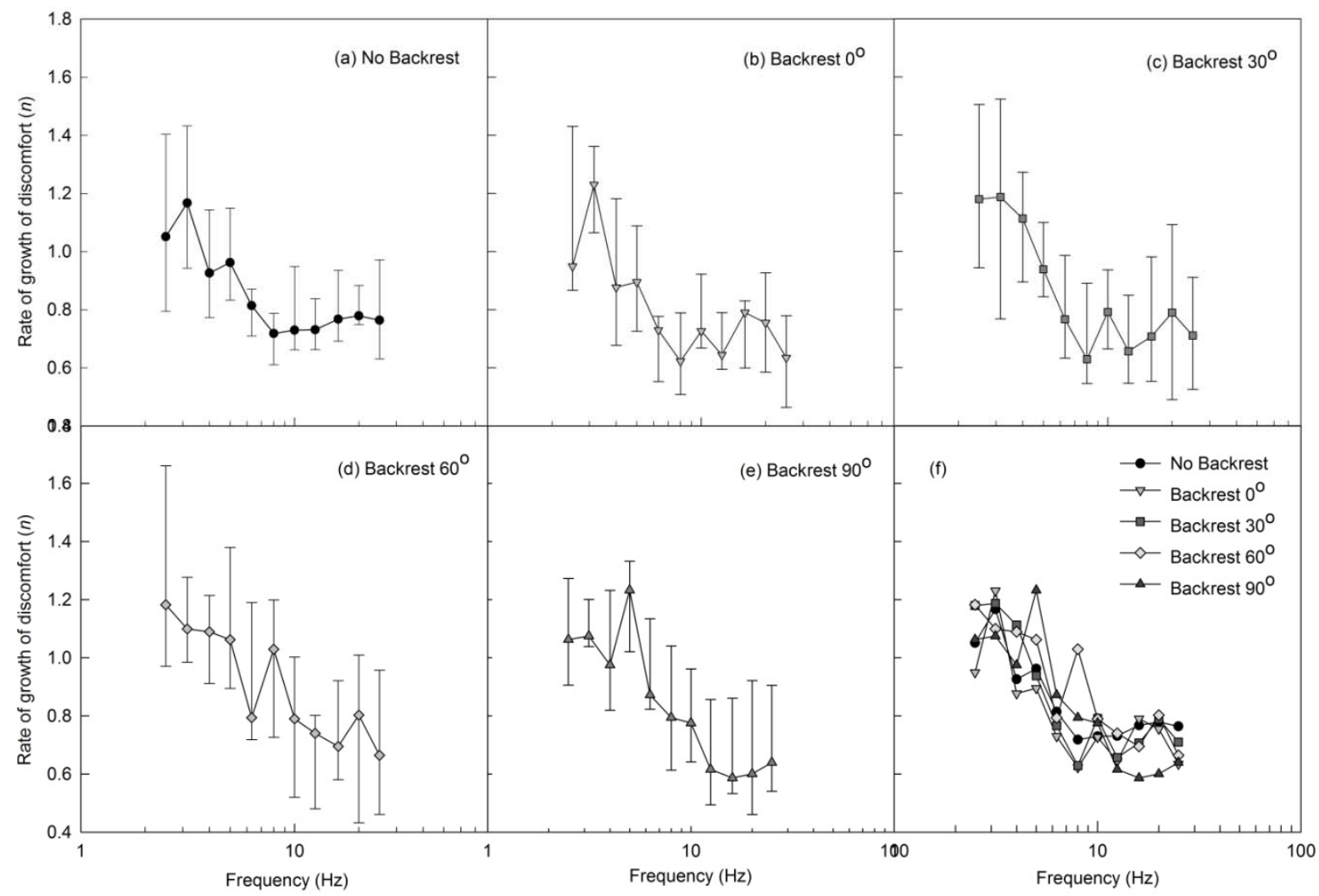




\section{FIGURE 4}
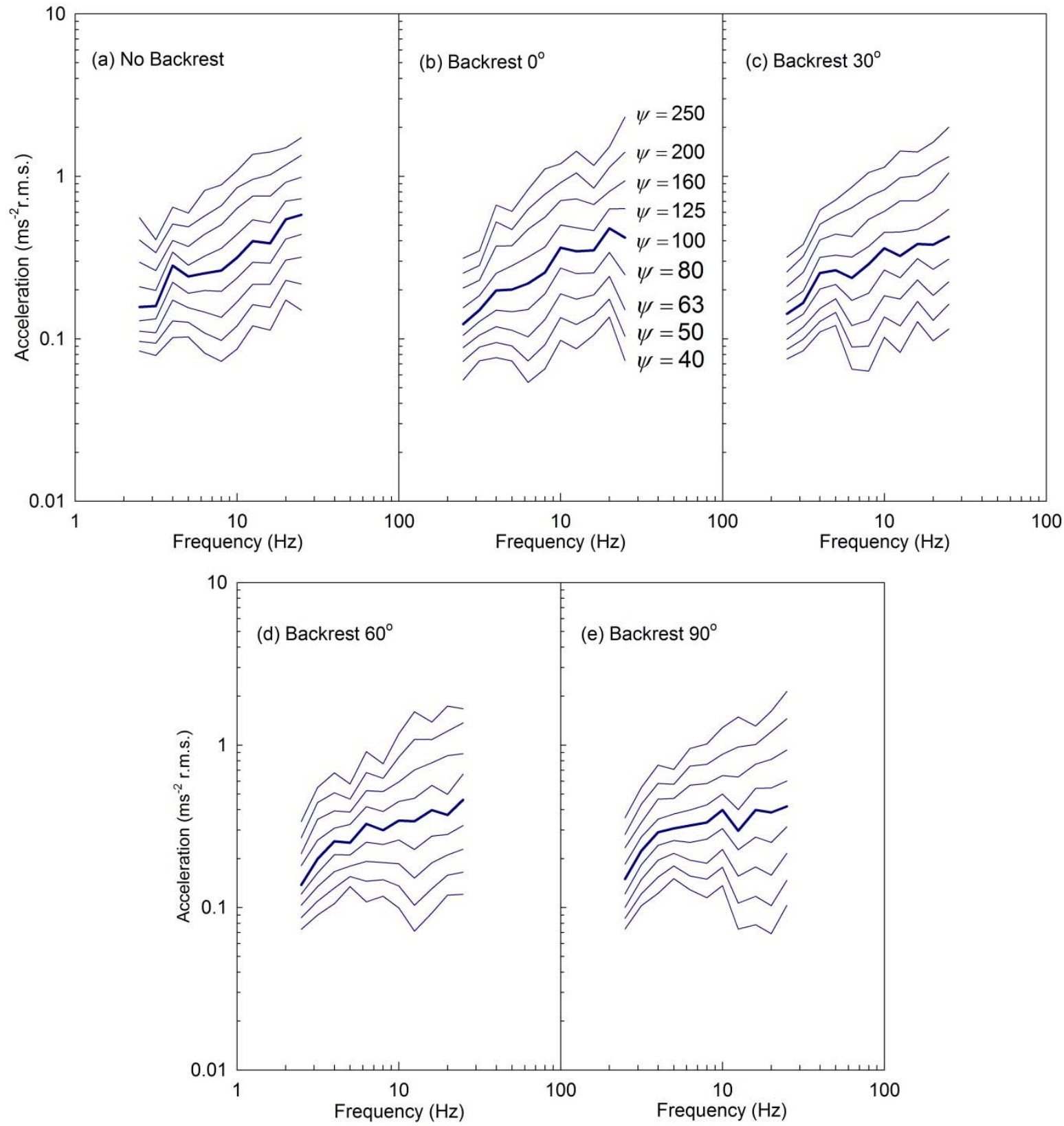
FIGURE 5
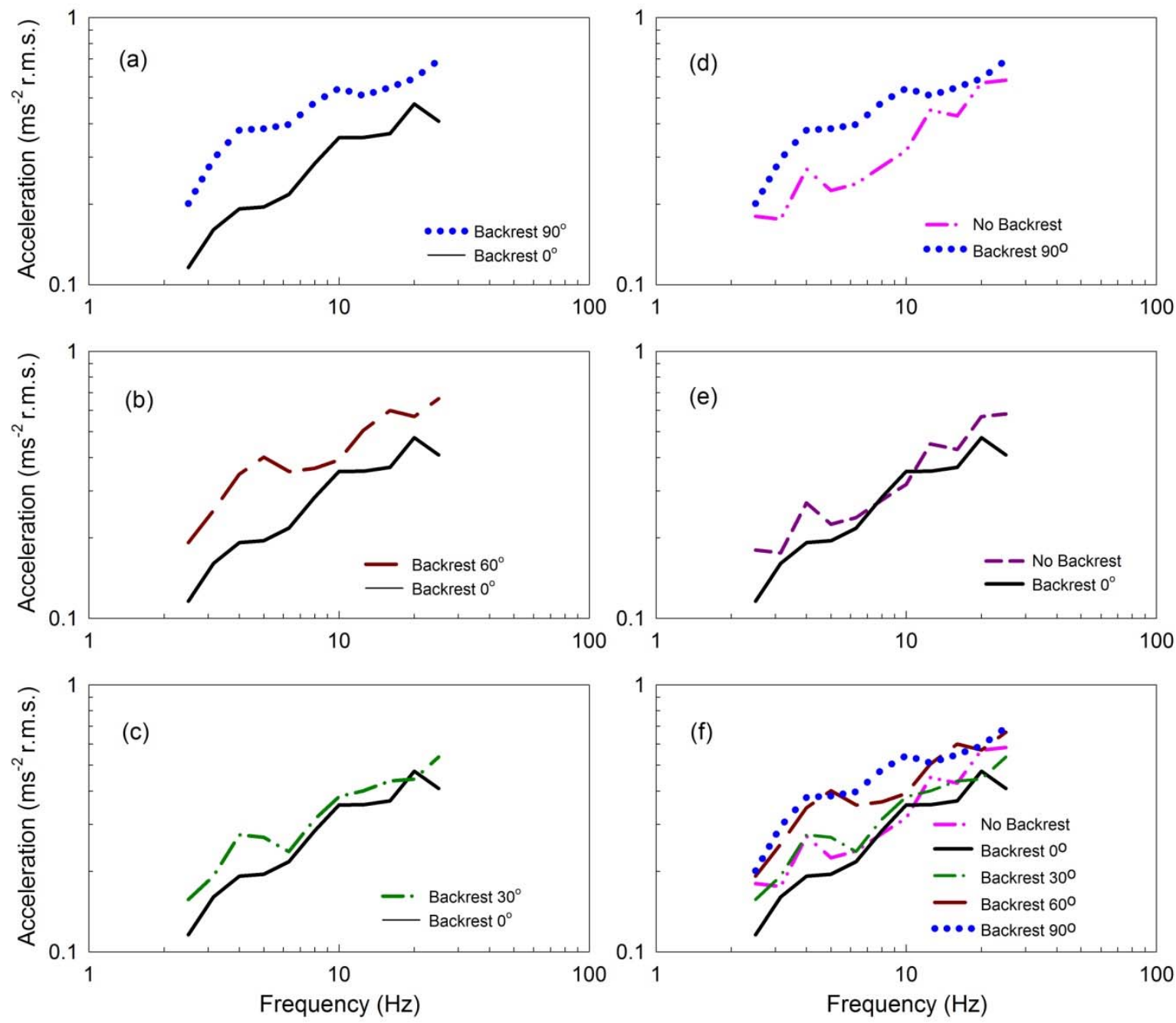


\section{FIGURE 6}
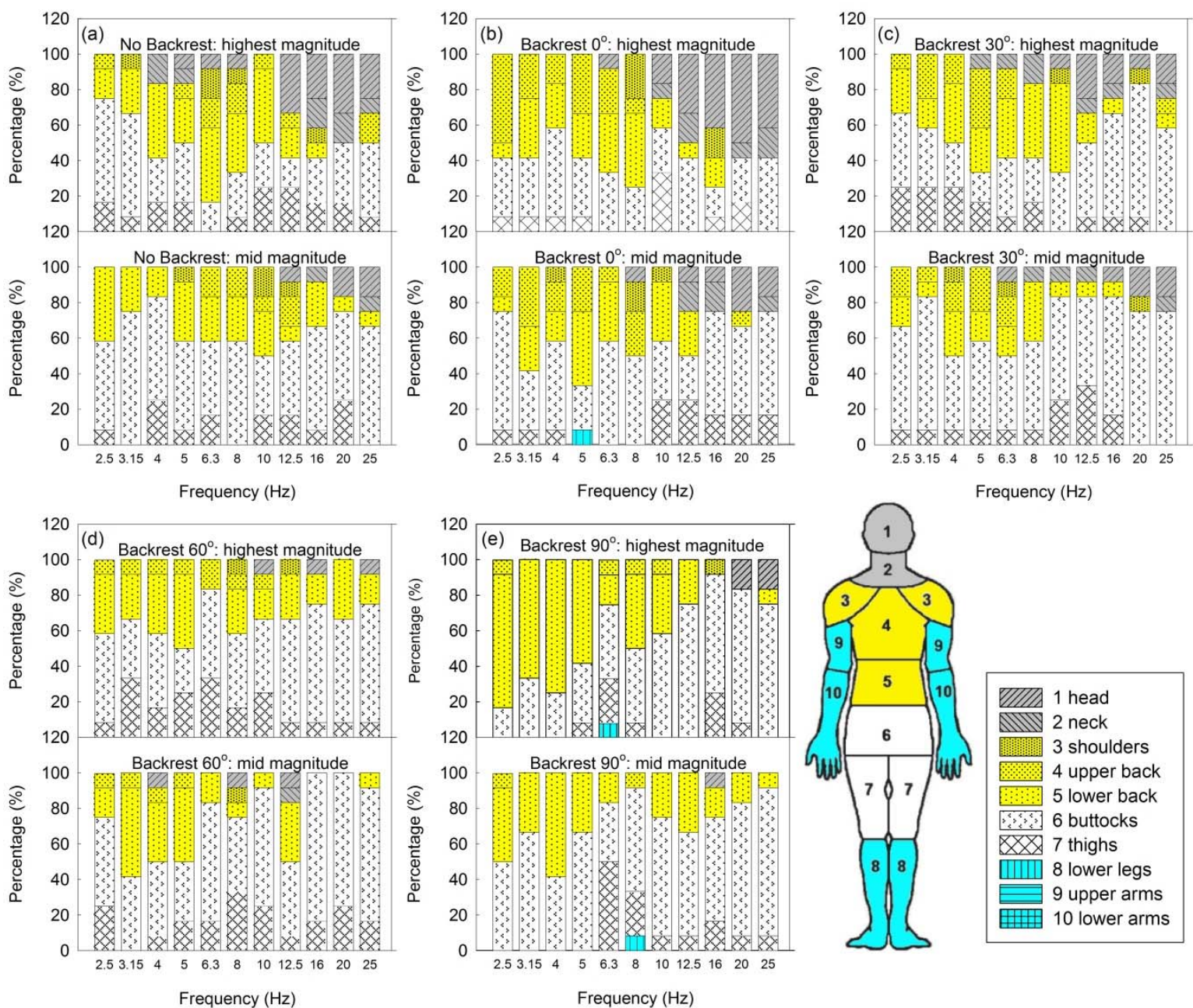

Frequency $(\mathrm{Hz})$

Frequency $(\mathrm{Hz})$ 


\section{FIGURE 7}

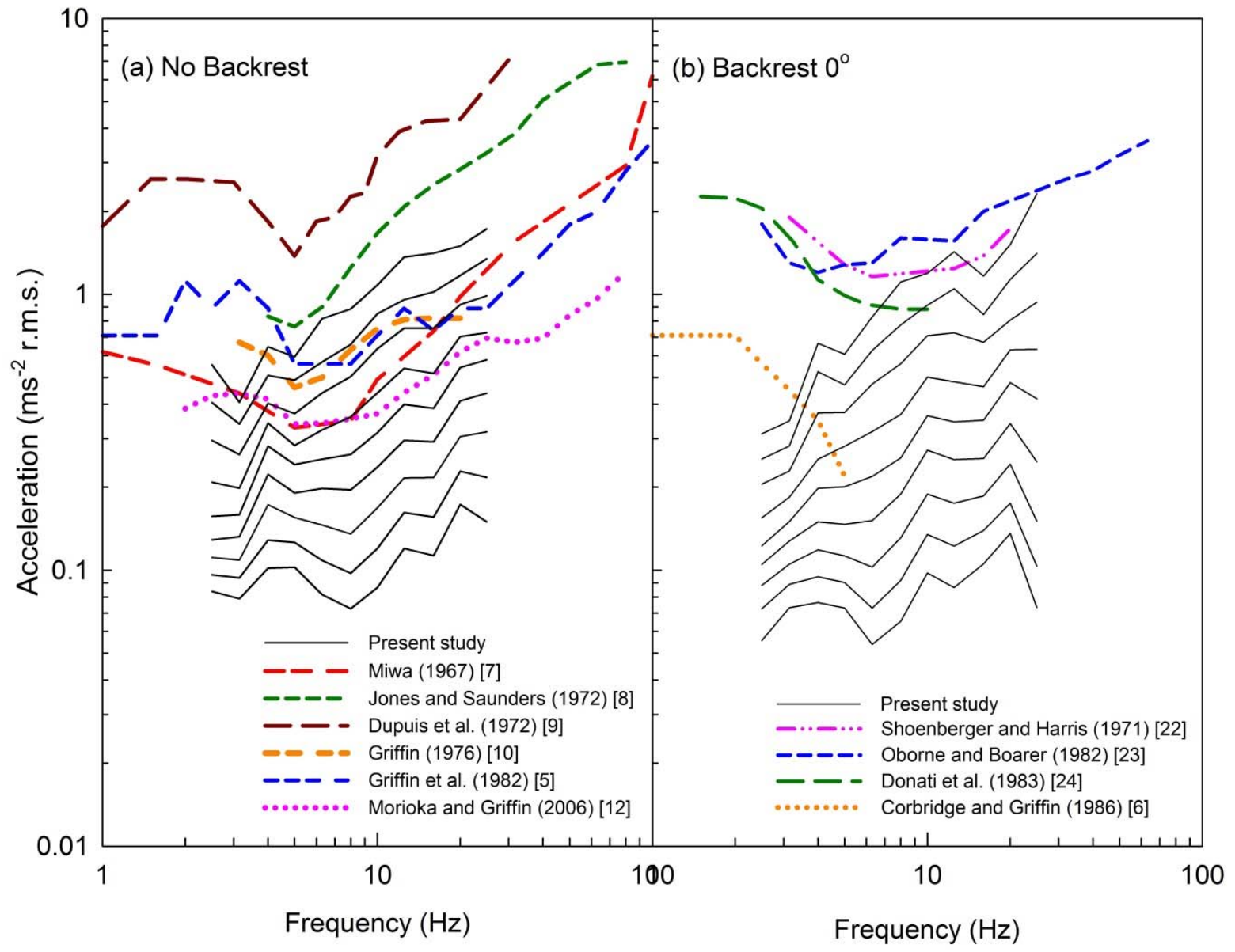


FIGURE 8

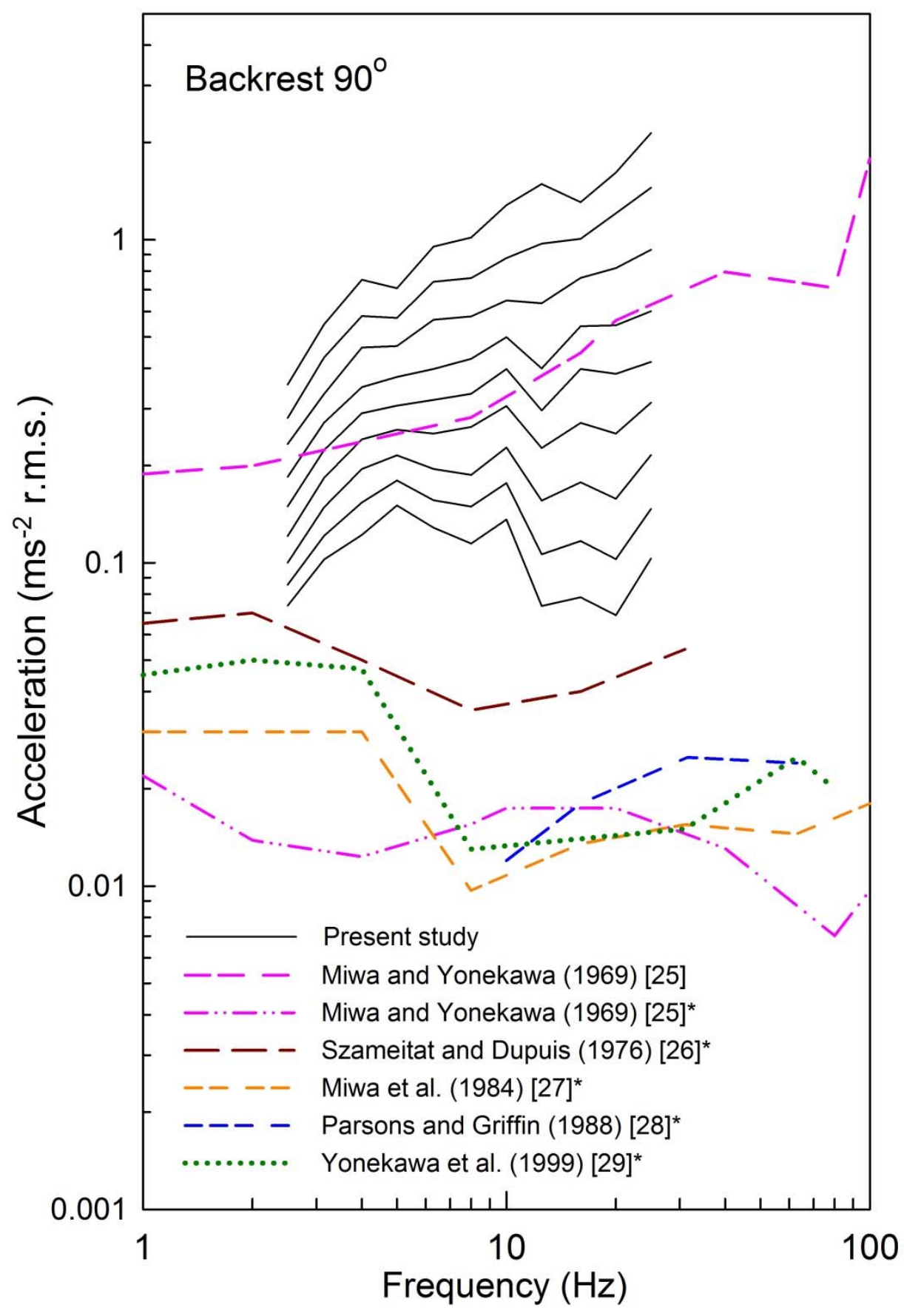


Equivalent comfort contours for vertical seat vibration: effect of vibration magnitude and backrest inclination Basri, B. \& Griffin, M. J. 26 Apr 2012 In : Ergonomics. 55, 8, p. 909-922.

\section{FIGURE 9}

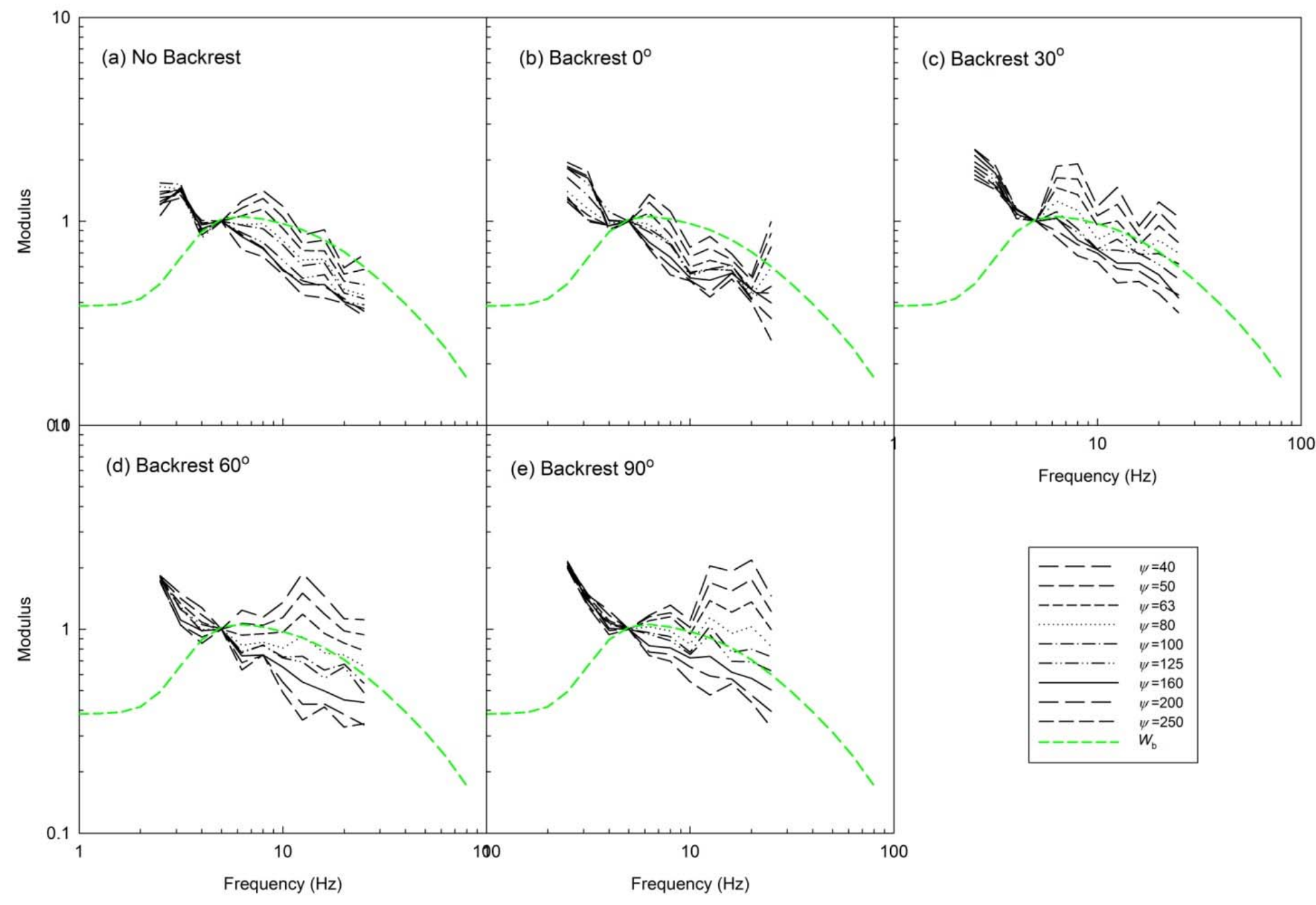


TABLE 1 Median exponents, $n$, and constants, $k$, for vertical seat vibration with no backrest, and with stationary backrest inclined at $0^{\circ}$ (upright), $30^{\circ}, 60^{\circ}$ and $90^{\circ}$ (recumbent).

\begin{tabular}{|c|c|c|c|c|c|c|c|c|c|c|}
\hline \multirow{2}{*}{ Frequency } & \multicolumn{5}{|c|}{ Exponent $(n)$} & \multicolumn{5}{|c|}{ Constant $(k)$} \\
\hline & No Backrest & $0^{\circ}$ & $30^{\circ}$ & $60^{\circ}$ & $90^{\circ}$ & No Backrest & $0^{\circ}$ & $30^{\circ}$ & $60^{\circ}$ & $90^{\circ}$ \\
\hline 2.5 & 1.05 & 0.95 & 1.18 & 1.18 & 1.06 & 420.8 & 796.1 & 969.5 & 986.6 & 745.3 \\
\hline 3.15 & 1.17 & 1.23 & 1.19 & 1.10 & 1.07 & 825.8 & 851.0 & 896.6 & 483.0 & 477.5 \\
\hline 4 & 0.93 & 0.88 & 1.11 & 1.09 & 0.98 & 374.3 & 359.2 & 418.9 & 359.6 & 319.4 \\
\hline 5 & 0.96 & 0.89 & 0.94 & 1.06 & 1.23 & 422.3 & 402.2 & 361.5 & 433.7 & 373.9 \\
\hline 6.3 & 0.81 & 0.73 & 0.77 & 0.79 & 0.87 & 295.4 & 286.6 & 289.0 & 266.3 & 262.5 \\
\hline 8 & 0.72 & 0.62 & 0.63 & 1.03 & 0.79 & 273.9 & 234.9 & 242.5 & 328.2 & 247.7 \\
\hline 10 & 0.73 & 0.73 & 0.79 & 0.79 & 0.78 & 229.7 & 215.8 & 240.4 & 227.6 & 218.8 \\
\hline 12.5 & 0.73 & 0.64 & 0.66 & 0.74 & 0.62 & 206.0 & 194.2 & 204.4 & 193.1 & 206.3 \\
\hline 16 & 0.77 & 0.79 & 0.71 & 0.69 & 0.59 & 198.5 & 225.9 & 198.4 & 184.7 & 198.9 \\
\hline 20 & 0.78 & 0.75 & 0.79 & 0.80 & 0.60 & 172.4 & 186.2 & 178.4 & 178.3 & 185.4 \\
\hline 25 & 0.76 & 0.63 & 0.71 & 0.66 & 0.64 & 162.8 & 169.4 & 153.7 & 170.2 & 169.3 \\
\hline
\end{tabular}

